\title{
Traditional Chinese Herbs Improve Salivation and Frequent Nighttime Urination in Patients with Amyotrophic Lateral Sclerosis
}

\author{
Penglin Gao Weilong Liao Chuanhe Sun Wenfei Jiang Weidong Pan Te Liu
}

Department of Neurology, Shuguang Hospital Affiliated to Shanghai University of Traditional

Chinese Medicine, Shanghai, China

\section{Keywords}

Amyloid lateral sclerosis - Traditional Chinese medicine - Salivation · Frequent nighttime urination · Jian-Pi Lian-Se Tang · Epworth Sleepiness Scale · Amyotrophic Lateral Sclerosis Function Rating Scale

\section{Abstract}

Aims: The aim of this study was to explore the efficacy of Jian-Pi Lian-Se Tang (JPLST), a formula of traditional Chinese medicine for the treatment of salivation and frequent nighttime urination (FNU) in patients with amyotrophic lateral sclerosis (ALS). Method: Forty-eight patients with ALS suffering from salivation and/or FNU who were outpatients or inpatients of Shuguang Hospital were included into the study and divided into 2 groups as follows: the JPLST group $(n=24)$ included patients who were additionally treated with JPLST, and the control group $(n=24)$ included patients who were treated by routine Western medicine (for 6 weeks). The changes in the quantity of salivation/sialorrhea (QS) (in $\mathrm{mL}$ ) and in FNU as the primary result and the total scores of the Amyloid Lateral Sclerosis Function Rating Scale (ALSFRS) and of the Epworth Sleepiness Scale (ESS) as the secondary result were used to evaluate the clinical efficacy for both groups. Results: No significant differences were found for the baseline of QS, FNU, and the scores of the ESS and of the ALSFRS before and after treatment for both groups. At the end of the 6th week, 2 patients in the JPLST group and 4 patients in the control group withdrew from the study. QS and FNU were much less severe in the JPLST group than before treatment, and the ESS scores were improved, too. The ALSFRS did not show any significant differences in both groups compared with before treatment at the end of week 6 . No side effects were found for both groups by laboratory tests. Conclusion: The much improved parameters of QS, FNU, and ESS for the JPLST group indicate that JPLST may be a potential additional treatment for salivation and FNU in ALS patients. Largescale multicenter double-blind randomized-control studies are needed to verify the effectiveness of JPLST in improving salivation and FNU in patients with ALS. @ 2017 The Author(s)

Dr. Weidong Pan

Department of Neurology, Shuguang Hospital Affiliated to Shanghai University of Traditional Chinese Medicine

528, Zhang-Heng Road, Pu-Dong New Area, Shanghai 201203 (China)

E-Mail panwd @ medmail.com.cn
Dr. Te Liu, Shanghai Geriatric Institute of Chinese Medicine Longhua Hospital

Shanghai University of Traditional Chinese Medicine, Building C 365 Xiangyang Road, Shanghai 200031 (China)

E-Mail teliu79@126.com 


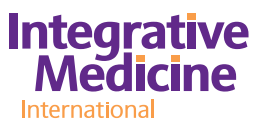

International

\section{Background}

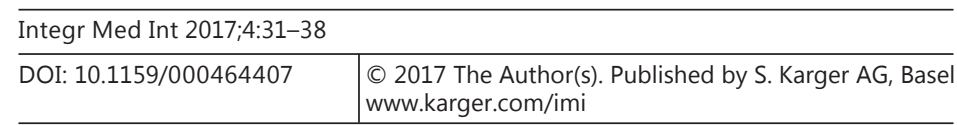

Gao et al.: Traditional Chinese Herbs Improve Salivation and Frequent Nighttime Urination in Patients with Amyotrophic Lateral Sclerosis

Amyotrophic lateral sclerosis (ALS) is a rare, rapidly progressive, and fatal disease that causes muscle weakness and atrophy throughout the body, and patients with ALS ultimately lose all voluntary movement [1]. ALS is diagnosed as "flaccidity syndrome" by traditional Chinese theory based on the weakness and atrophy of the limbs and body, and most patients are eventually unable to use their hands and arms and have difficulty with chewing, swallowing, and breathing [2]. Most traditional Chinese doctors believe that the pathogenesis of motor neuron degeneration in ALS has its origin in a deficiency in the spleen, which is the organ that controls the creation of muscle, or a deficiency of the spleen accompanied by excess consumption [3]. In addition to weakness and muscle atrophy, there are some other symptoms which influence the quality of daily life of patients very much. Salivation and nighttime urination are 2 important symptoms and may disrupt the quality of life to a greater extent than other disorders of the patients. Both frequent nighttime urination (FNU) and sialorrhea are explained by the theory of traditional Chinese medicine (TCM) in terms of dysfunction of body fluid control. TCM theory considers these symptoms to be a Qi deficiency in the spleen and kidney [4]. We used the TCM herbs of Jian-Pi Lian-Se Tang (JPLST) which can improve the Qi function of the body and showed that JPLST improved astringency function after 6 weeks of treatment in patients who suffered from salivation and FNU compared to patients not treated with TCM.

\section{Subjects and Methods}

\section{Subjects}

Sixty-five patients with ALS were assessed for eligibility. Finally, 48 patients defined as having probable or definite ALS according to the El Escorial criteria [5] and diagnosed at the Department of Neurology of Shuguang Hospital Affiliated to Shanghai University of TCM were invited to participate in the study (Fig. 1). The age of the patients ranged from 37 to 80 years (50.4 \pm 6.7$)$. The baseline clinical characteristics of the 2 ALS groups, including age, gender, mean symptom duration at baseline in months, mean time from diagnosis to baseline in months, treatment with Riluzole tablets (Sanofi-Aventis Co., Ltd., France), and mean Amyloid Lateral Sclerosis Function Rating Scale (ALSFRS) [6] scores at baseline are presented in Table 1. Patients were included in the study if they suffered at least from sialorrhea $(\geq 15 \mathrm{~mL}$ daily) and/or from FNU ( $\geq 3$ times per night). Patients who suffered from other symptoms or diseases which may influence the quality of sleep or the quantitative evaluation of sialorrhea were excluded from the study, i.e., patients with dementia, chronic obstructive pulmonary disease, obstructive sleep apnea/hypopnea syndrome, pain symptom, alcohol or drug addiction, angina pectoris, stroke, bell palsy (facial paralysis), mumps, and restless leg syndrome. In addition, in order to evaluate the whole quality of sleep (especially to assess daytime sleepiness), outcome measures on quality of life were recorded using the Epworth Sleepiness Scale (ESS) [7].

All patients underwent a neurologic examination and routine blood tests (including serum iron and ferritin, $\mathrm{B}_{12}$ vitamin, and folate concentrations). Patients with any abnormality in the above-mentioned tests or with an apnea-hypopnea index $>5$ were also excluded.

\section{Randomization, Masking, and Drug Administration}

An unblinded pharmacist generated randomization codes using an Excel (Microsoft Office) random number generator (Microsoft, USA) in blocks of 2 and 4 participants. Kits were given sequential numbers that corresponded to the randomization key and were maintained 
Gao et al.: Traditional Chinese Herbs Improve Salivation and Frequent Nighttime Urination in Patients with Amyotrophic Lateral Sclerosis

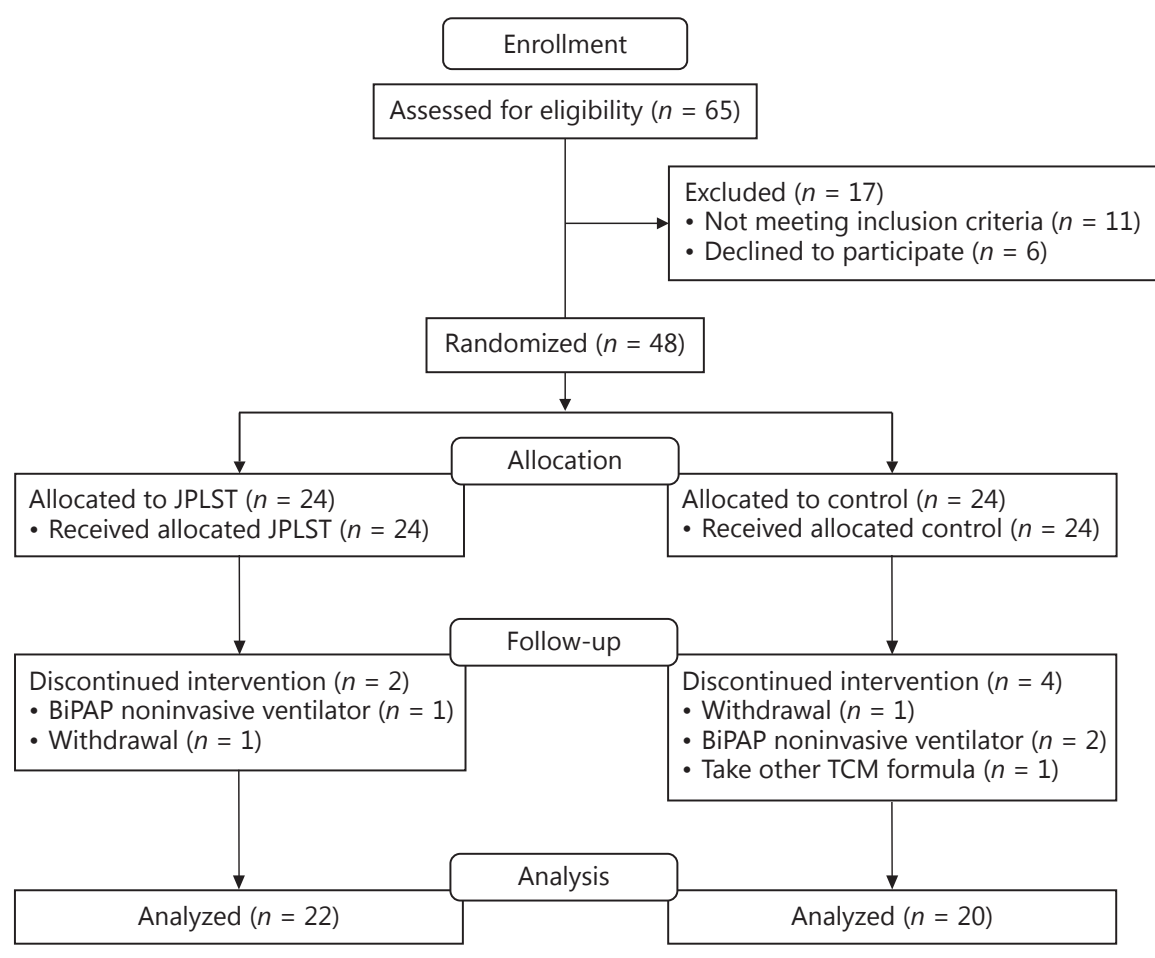

Fig. 1. CONSORT flow diagram of JPLST decoction for the treatment of patients with ALS. ALS, amyotrophic lateral sclerosis; JPLST, Jian-Pi Lian-Se Tang; BiPAP, biphasic positive airway pressure; TCM, traditional Chinese medicine.

Table 1. The baseline clinical characteristics of the 2 ALS groups

\begin{tabular}{lccc}
\hline Subjects & JPLST group $(n=22)$ & Control group $(n=20)$ & $p$ value \\
\hline Age, years & $50.2 \pm 7.2$ & $50.7 \pm 4.2$ & 0.47 \\
Men/women & $14 / 8$ & $13 / 7$ & - \\
Disease duration, months & $23.9 \pm 18.6$ & $24.3 \pm 17.6$ & 0.25 \\
Mean time from diagnosis, months & $17.35 \pm 11.86$ & $16.95 \pm 12.51$ & 0.18 \\
Riluzole tablets, mg/day & $87.36 \pm 9.59(n=12)$ & $89.49 \pm 8.36(n=13)$ & 0.37 \\
First developed ALS in & & & \\
$\quad$ limbs/bulbar/both & $18 / 3 / 3$ & $19 / 2 / 3$ & - \\
Epworth Sleepiness Scale & $9.17 \pm 2.02$ & $9.12 \pm 1.98$ & 0.17 \\
ALS Function Rating Scale & $33.1 \pm 5.6$ & $34.4 \pm 6.1$ & 0.65
\end{tabular}

Values are means \pm standard deviations unless otherwise indicated. ALS, amyotrophic lateral sclerosis.

in a secure location. When randomized, each successive participant was assigned by an electronic Clinical Trial Management System to the next numbered kit in sequence at each site. The ALS patients were randomized into either the JPLST group $(n=24)$ or the control group $(n=24)$. There was no stratification of patients according to the onset region, age, or respiratory function since all patients enrolled were supposed to receive both treatments. 
Gao et al.: Traditional Chinese Herbs Improve Salivation and Frequent Nighttime Urination in Patients with Amyotrophic Lateral Sclerosis

The patients in the treatment group received the JPLST decoction (Diaphragma juglandis 9 g, Radix Astragali 30 g, Codonopsis pilosula 15 g, Poria cocos 9 g, roasted Rhizoma Atractylodis Macrocephalae 9 g, Glycyrrhiza 9 g, Rosa laevigata $12 \mathrm{~g}$, and corn stigma 9 g). All herbs were placed in $400 \mathrm{~mL}$ of cold water, soaked for $30 \mathrm{~min}$, and boiled for $30 \mathrm{~min}$ using a small flame to obtain about $100 \mathrm{~mL}$ of decoction. The decoctions were prepared by the manufacturing laboratory of Shuguang Hospital $(50 \mathrm{~mL})$ twice per day. The other patients were treated with routine treatment according to the guideline of China [8]. They were not treated by any other complementary and/or alternative treatments, such as other TCM, massage, or acupuncture.

\section{Clinical Efficacy and Safety Evaluation}

A detailed history and neurological examination were performed 3 times by a neurologist in all subjects at baseline (before treatment) and at the end of weeks 2 and 6 throughout the 6-week study period. In order to determine the quantity of sialorrhea (QS) in the patients, the patients or their caregivers were trained by nurses to estimate the QS using towels, small cups, tissues, etc. The average of 2 days of sialorrhea was the primary outcome for evaluating the severity of sialorrhea. The frequency of nighttime urination was counted to assess the changes in overnight urine output. We used the improvement in QS and FNU as the primary result and the ALSFRS [6] and the ESS as the secondary result to evaluate the efficacy of the additional treatment. Standard laboratory tests, including red blood cell count, chemistry, and renal and liver function, were performed at baseline and at the posttreatment discontinuation visit. Safety was evaluated as the incidence and severity of adverse events, and their relationship to treatment was determined based on the results of the laboratory tests, patient reports, and the judgment of the investigators.

\section{Statistical Analysis}

Repeated-measures ANOVA was conducted to test the differences in changes in outcomes at baseline and at the end of weeks 2 and 6 for both groups. Differences at baseline between the JPLST group and the control group were analyzed using the $t$ test. A significant difference was defined as $p<0.05$. SPSS (Windows version 17.0) software was used for statistical analyses. All data are expressed as means \pm standard deviations.

\section{Statement of Ethics}

Signed informed consent was obtained from the patients before participation. The study was approved by the Ethics Committee of Shuguang Hospital Affiliated to Shanghai University of TCM and was performed in accordance with the principles outlined in the Declaration of Helsinki.

\section{Results}

At the end of the 6th week, 2 patients in the JPLST group withdrew from the study. One patient started using the biphasic positive airway pressure (BiPAP) noninvasive ventilator, and another patient withdrew from the trial without any reason. Four patients in the control group withdrew from the study. One of these patients discontinued without any reason, 2 patients started using the BiPAP noninvasive ventilator, and another started taking TCM to treat another uncomfortable symptom (Fig. 1). No significant differences were found in the baseline values of QS and FNU and in the ESS and ALSFRS scores between the 2 groups (Table 1). 
Fig. 2. Changes in the quantity of saliva (a) and the frequency of nighttime urination (b) before and at week 6 of the TCM treatment. c, d Changes in the Amyotrophic Lateral Sclerosis Function Rating Scale (ALSFRS) and the mean of the Epworth Sleepiness Scale in the 2 groups of patients. * $p<0.05$, compared to before treatment for the Jian-Pi Lian-Se Tang group. ${ }^{+} p<0.05$, compared to the control group.

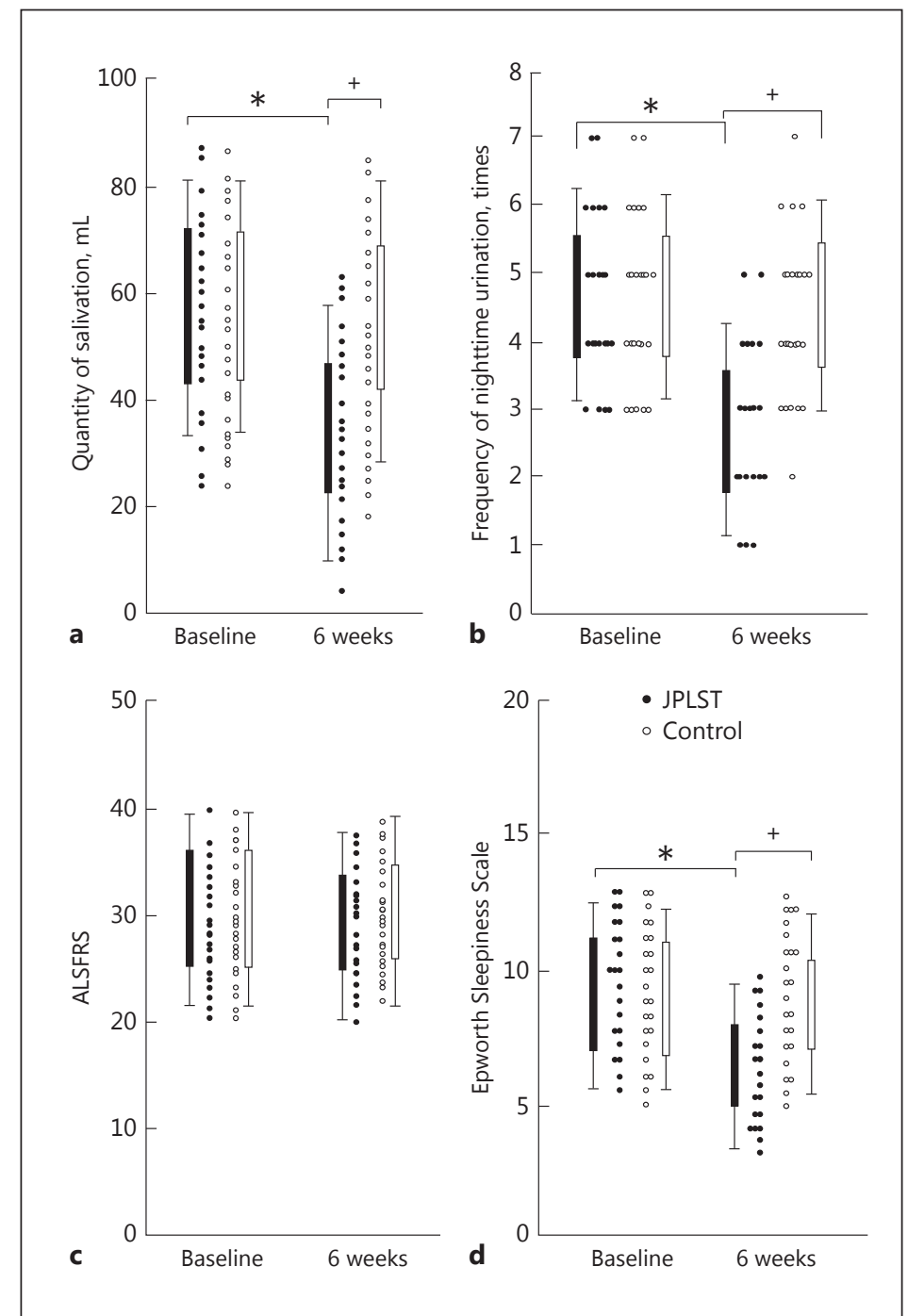

The primary outcome were the changed QS and FNU values of the patients in the JPLST group. They were superior to those in the control group at the end of week 6 (Fig. 2a, b, d). The total score of the ALSFRS indicated no such differences between the 2 groups during the investigation (Fig. 2c). The ESS was $6.33 \pm 2.16$ in the JPLST and $8.51 \pm 2.02$ in the control group (Fig. 2d), and the difference was significant ( $p<0.044)$ at the end of week 6 . There were no abnormal laboratory test result values in either group.

\section{Discussion}

After Prof. Tu Youyou was awarded the Nobel Prize for Medicine and Physiology in malaria research [9], TCM has been getting more attractive for the treatment of difficult diseases which cannot be improved or treated by Western medicine. ALS is a fatal neurodegenerative disorder characterized by progressive degeneration of motor neurons, leading to paralysis and death, typically within 3-5 years from symptom onset. Riluzole is the only FDAapproved "orphan drug" for ALS and, using the BiPAP noninvasive ventilator, prolongs life by 
Gao et al.: Traditional Chinese Herbs Improve Salivation and Frequent Nighttime Urination in Patients with Amyotrophic Lateral Sclerosis

only 2-3 months in patients treated for at least 18 months [10]. It did not demonstrate any benefit for improving such painful symptoms as sialorrhea and FNU. These 2 symptoms of ALS patients lack effective treatments by Western medicine. In this study, we demonstrate that JPLST, a TCM, ameliorates the 2 symptoms using the parameters of QS and FNU together with the conventional ESS and ALSFRS scores as evaluation methods.

We previously demonstrated that the use of TCM could improve some symptoms of patients with neurodegenerative diseases [11-14]. Sialorrhea and FNU are 2 liquidcontrolling problems in TCM theory; the liquid-controlling power is TCM Qi, which comes from the TCM viscera functions of spleen and kidney of the body [15]. All neurodegenerative diseases, including Parkinson disease, Alzheimer disease, and ALS, have been considered as pro-senescence diseases in TCM [16]. The kidney has been regarded as the initial essence and basic power source of the body, controlling the velocity of all growth, development, and senescence of the body [17]. If pro-senescence starts at an early age, neurodegenerative diseases will occur. The kidney also demonstrates the switch function for controlling body liquids, such as saliva and urine, especially controlling their opening time and excretion quantity according to TCM theory. The traditional Chinese spleen is 1 resource of a posteriori power; it can consecutively absorb nutrition and essence to keep up the metabolism of the body [18]. The basic essence and energy of Qi come from the kidney, and the kidney is also the foundation of the spleen [19]. The other important function of the spleen is that it can dredge the liquid vessels of the body, keeping all the body liquids in circulation in the liquid vessels (it is similar to a "blood vessel" in TCM, but it cannot be seen as a blood vessel in Western medicine). If the function of the liquid circulation of the TCM spleen is getting worse (Qi deficiency), the liquids, such as saliva and urine, may go anywhere at any time. The TCM liver is a coordinator; it can coordinate all the functions of the viscus, including the kidney and the spleen. According to TCM, ALS can be explained as the pro-senescence of the kidney, and the causes of sialorrhea and FNU are due to the weak functions of the kidney, spleen, and liver with its Qi deficiency.

JPLST contains 8 components of TCM herbs. Diaphragma juglandis (枫心木) is the most important herb in TCM and is taken from the walnut; it induces a very powerful increase in the function (as Qi) of the TCM kidney (tonifying kidney function), and it also contributes to a stronger astringency function in many clinical and basic studies, which can reduce the secretion of urine and saliva [20]. Radix Astragali (黄芪) can supply powerful Qi to increase the nourishing functions of the decoction [21]. Codonopsis pilosula (党参) is an important herb which can nourish the Qi of the TCM spleen and tonify the Qi of the kidney. Many modern researchers indicated that it could also significantly enhance the immune-enhancing activity in the nonspecific immune response [22]. Poria cocos (获苓) is a liquid-modifying herb which can control the secretion of normal liquid and the excretion of metabolic liquid according to TCM theory. Roasted Rhizoma Atractylodis Macrocephalae (炒白术) and Glycyrrhiza deserticola (甘草) are 2 tonifying spleen herbs which can increase the Qi of the body, improving the function of astringency (circulation of TCM Qi), and can also improve the digestive system and atrophy of patients with ALS [23]. It shows powerful effects by increasing the function of the kidney, supplying power to the kidney, modifying urine timing and quantity, and decreasing the abnormal secretion of saliva. Rosa laevigata (金樱子) and corn stigma (玉米须) can increase the control power of urine and saliva and stop the secretion of abnormal liquids of the body in TCM function. Glycyrrhiza deserticola (甘草) can coordinate all the herbs so that the 8 herbs work together accurately to control the urine and saliva problem properly and decrease the potential adverse effects of these herbs.

The present study indicates that JPLST did not improve the total score of the ALSFRS, but it did improve QS and FNU parameters as well as the ESS scores in the treatment group. Improvement in FNU was clearly demonstrated in the profile of actigraphic recording scores 
Gao et al.: Traditional Chinese Herbs Improve Salivation and Frequent Nighttime

Urination in Patients with Amyotrophic Lateral Sclerosis

(Fig. 1, Fig. 2b, c). Sleep disturbance, which is frequent among patients with ALS, is thought to be due to the disruption by FNU.

Our study has some limitations. Firstly, the placebo granule used in this trial contains 5 component herbs as placebo, but these herbs do have functions in TCM, although the influence is very weak. Secondly, the method for assessing QS by patients or caretakers was subjective, so that it does not accurately reflect the changes in QS. Thirdly, this was not a randomized controlled trial, and insufficient cases were included, which are other shortcomings of our study. In order to validate the causes of the disease based on clinical data, large-scale multicenter double-blind randomized-control studies are needed to verify the effectiveness of LSF for the treatment of sialorrhea and FNU in patients with ALS. JPLST is well tolerated in longterm administration, has no intolerable adverse effects, and hence is likely a suitable choice as an additional drug to improve the sialorrhea and FNU symptoms of ALS.

\section{Acknowledgements}

This study was sponsored and supported by the National Natural Science Foundation of China (81373619).

\section{References}

1 Tandan R, Bradley WG: Amyotrophic lateral sclerosis: part 1. Clinical features, pathology, and ethical issues in management. Ann Neurol 1985;18:271-280.

2 Pan W, Su X, Bao J, Wang J, et al: Open randomized clinical trial on JWSJZ decoction for the treatment of ALS patients. Evid Based Complement Alternat Med 2013, DOI: 10.1155/2013/347525.

-3 Chen X, Pan W: The treatment strategies for neurodegenerative diseases by integrative medicine. Integr Med Int 2014;1:223-225.

4 Pan W, Chen X, Bao J, Bai Y, et al: The use of integrative therapies in patients with amyotrophic lateral sclerosis in Shanghai, China. Evid Based Complement Alternat Med 2013, DOI: 10.1155/2013/613596.

5 Mitchell JD, Borasio GD: Amyotrophic lateral sclerosis. Lancet 2007;369:2031-2041.

-6 Cedarbaum JM, Stambler N, Malta E, Fuller C, Hilt D, Thurmond B, Nakanishi A: The ALSFRS-R: a revised ALS functional rating scale that incorporates assessments of respiratory function. BDNF ALS Study Group (phase III). J Neurol Sci 1999;169:13-21.

7 Benbadis SR, Mascha E, Perry MC, Wolgamuth BR, et al: Association between the Epworth Sleepiness Scale and the multiple sleep latency test in a clinical population. Ann Intern Med 1999;130:289-292.

8 Huang H: The development of integrative therapies in patients with amyotrophic lateral sclerosis. Guo Ji Shen Jing Bing Xue Shen Jing Wai Ke Za Zhi 2006;33:322-326.

$\$ 9$ Litscher D, Litscher G: China's Tu Youyou, Nobel Laureate in Medicine 2015, and relationships with the TCM Research Center Graz at the Medical University. Integr Med Int 2016;3:1-9.

10 Geevasinga N, Menon P, Ng K, Van Den Bos M, et al: Riluzole exerts transient modulating effects on cortical and axonal hyperexcitability in ALS. Amyotroph Lateral Scler Frontotemporal Degener 2016;17:580-588.

11 Pan W, Yang X, Zhou H: The development of integrative medicine. Integr Med Int 2016;3:96-98.

12 Pan W, Kwak S, Liu Y, Fang Z, et al: A compound belonging to traditional Chinese medicine improves nocturnal activity in Parkinson's disease. Sleep Med 2011;12:307-308.

13 Pan W, Wang Q, Kwak S, Song Y, et al: Shen-Zhi-Ling oral liquid improves behavioral and psychological symptoms of dementia in Alzheimer's disease. Evid Based Complement Alternat Med 2014, DOI: 10.1155/2014/913687.

14 Pan W, Kwak S, Liu Y, Sun Y, Fang Z, Qin B, Yamamoto Y: Traditional Chinese medicine improves activities of daily living in Parkinson's disease. Parkinsons Dis 2011, DOI: 10.4061/2011/789506.

15 Huang KF, Tang ST, Chuang CY, Han WR, Lin JH, Young ST: Different patterns of dynamic variations on electrical conductances of acupoints between Qi vacuity and Qi non-vacuity after glucose ingestion. J Altern Complement Med 2011;17:843-849.

16 Liu Q, Zhang P, Pan J, Li Z, et al: Cerebral activity changes in different traditional Chinese medicine patterns of psychogenic erectile dysfunction patients. Evid Based Complement Alternat Med 2015;2015:503536.

17 Yu B, Zhou C, Zhang J, Ling Y, et al: Latest study on the relationship between pathological process of inflammatory injury and the syndrome of spleen deficiency and fluid retention in Alzheimer's disease. Evid Based Complement Alternat Med 2014;2014:743541. 
Gao et al.: Traditional Chinese Herbs Improve Salivation and Frequent Nighttime Urination in Patients with Amyotrophic Lateral Sclerosis

18 Liao W, Dang C, Pan W: The brief theory of viscus and its manifestations in traditional Chinese medicine. Integr Med Int 2017;4:13-18.

19 Cui HZ, Wang LM, Zhao X, Liu YY, et al: Metabonomics-based study of clinical urine samples in suboptimal health with different syndromes. Evid Based Complement Alternat Med 2013;2013:509134.

20 Zhao H, Bai H, Jing Y, Li W, et al: A pair of taxifolin-3-0-arabinofuranoside isomers from Juglans regia L. Nat Prod Res 2017;31:945-950.

21 Wei X, Zhang J, Li J, Chen S: Astragalus mongholicus and Polygonum multiflorum's protective function against cyclophosphamide inhibitory effect on thymus. Am J Chin Med 2004;32:669-680.

-22 Qin T, Ren Z, Lin D, Song Y, et al: Effects of selenizing Codonopsis pilosula polysaccharide on macrophage modulatory activities. J Microbiol Biotechnol 2016;26:1358-1366.

23 Pan W, Kwak S, Wu C, Ma J, et al: Effects of "Jiawei Sijunzi decoction" on movement dysfunction of AR2 mice with amyotrophic lateral sclerosis. Shanghai Zhong Yi Yao Da Xue Xue Bao 2013;27:60-63. 